A MIXED FINITE VOLUME METHOD FOR ELLIPTIC PROBLEMS

By

Ilya D. Mishev

and

Qian-Yong Chen

IMA Preprint Series \# 2059

( August 2005)

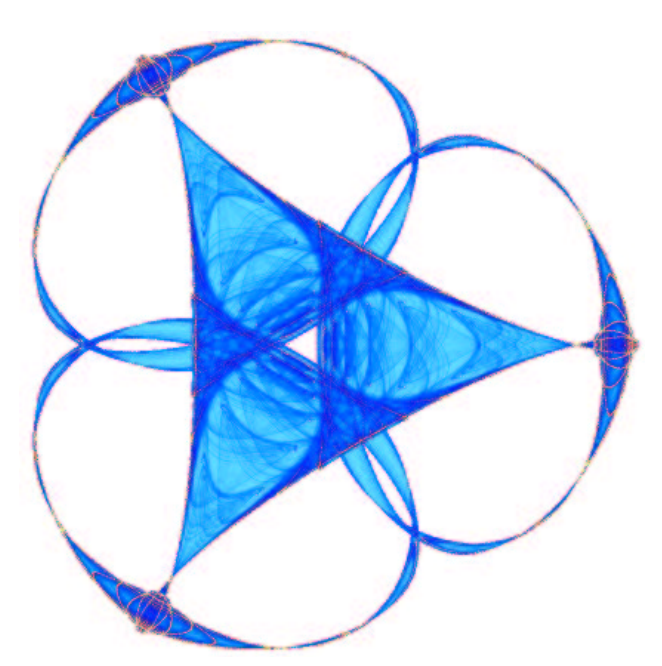

INSTITUTE FOR MATHEMATICS AND ITS APPLICATIONS

UNIVERSITY OF MINNESOTA 400 Lind Hall

207 Church Street S.E.

Minneapolis, Minnesota 55455-0436

Phone: 612/624-6066 Fax: 612/626-7370

URL: http://www.ima.umn.edu 


\title{
A Mixed Finite Volume Method for Elliptic Problems
}

\author{
Ilya D. Mishev and Qian-Yong Chen* \\ ExxonMobil Upstream Research Company \\ P.O. Box 2189, URC-URC-NW514 \\ Houston, TX 77252-2189
}

\begin{abstract}
We derive a novel finite volume method for the elliptic equation, using the framework of mixed finite element methods to discretize the pressure and velocities on two different grids (covolumes), triangular (tetrahedral) mesh and control volume mesh. The new discretization is defined for tensor diffusion coefficient and well suited for heterogeneous and anisotropic media. When the control volumes are created by connecting the mass center of each triangle to the midpoints of its edges, we show that the discretization is stable and first order accurate for both scalar and vector unknowns.
\end{abstract}

Key words; finite volume methods, cell-centered finite differences, error estimates AMS subject classification: 65N06, 65N12, 65N15.

${ }^{*}$ Postdoctoral associate at Institute for Mathematics and its Applications, University of Minnesota (qchen@ima.umn.edu). Partially funded by ExxonMobil Upstream Research Company. 


\section{Introduction}

Physical diffusion processes are usually modeled by elliptic equations. The heterogeneous diffusion tensor due to the heterogeneous anisotropic media imposes great challenges to the numerical schemes. There are several highly desirable properties of the discretization beyond the classical stability and accuracy, such as local mass conservation, discrete maximum principle, lack of grid orientation effects, etc, that are crucial in capturing the important details of the solutions of complicated (especially nonlinear) problems on relatively coarse grids.

In this paper we derive a method that is locally mass conservative and it can be applied to a general finite volume grid constructed from a given triangular or tetrahedral mesh. Typical example is the coupled Voronoi boxes (PEBI mesh) and Delaunay triangles (tetrahedra). When the control volume is formed by mass centers and edge midpoints of the triangles, we show the first order accuracy for the pressure gradient and the velocity. Our discretization is similar to the Finite Volume Element (FVE) method, but it produces direct approximation of the velocity which is not the case for FVE method. This can be very beneficial in some applications. In the proposed method, the velocity unknowns are eliminated and the resulting linear system for the pressure can be solved with the available solvers. Therefore, the estimated computational cost on triangular or tetrahedral mesh will be two to three times less than the cost for solving the same problem with the standard mixed finite element methods.

There have been extensive research on developing numerical schemes for the partial differential equations of interest on a given general grid. The classical finite difference schemes are only applicable to structured grids. The mimetic finite difference schemes proposed by Shashkov [31] are very promising in terms of dealing with highly distorted grids. The con-

vergence and super convergence $[6,7]$ are also established for smooth problems on smooth meshes by rewriting it into the form of mixed finite element methods. But the computational cost is an issue. 
Finite volume methods overcome most of the restrictions of finite difference schemes, and they are usually locally mass conservative. There have been a significant advance in the theory of the finite volume methods applied to diffusion equations with scalar coefficient on unstructured meshes $[2,17,21,23,29]$. Several methods for handling tensor coefficient have been proposed $[35,14,1]$. Recently the convergence is established for one of such methods, the multi-point flux approximation, on quadrilateral grids under the assumption of smooth mesh [19]. But a comprehensive theory for general mesh is still not available yet.

Control volume finite element methods, often called finite volume element methods are another worthy alternative $[5,15,11,30,16,24]$. They are applicable on flexible grids and are locally mass conservative, but do not preserve the symmetry of the differential operator, and do not produce direct approximation of the velocity field.

Mixed finite elements, since their introduction [27], have proven to be robust and give very accurate approximations. In some cases it is possible to reduce the system of coupled equations for the pressure and velocity to a system only for the pressure $[4,3]$, which is much easier to solve. Unfortunately some restrictions apply. There is considerable advance in designing methods for distorted general meshes $[22,10,33,34,12]$, but they are quite expensive and 3-D case is still under development $[25,20]$.

The proposed method in considerable extent alleviates the drawbacks of the known methods without significant increase of the computational cost.

The rest of the paper is organized as follows. In Sec. 2 we consider a simple model problem. The discretization is derived in Sec. 3. All necessary formulas for the implementation of the method are provided. Review of the theory for mixed finite element methods is outlined in Sec. 4 and theoretical estimates are proven in Sec. 5. Some numerical results are presented in Sec. 6. Finally we summarize the paper in the conclusions. 


\section{Model Problem}

Consider a model boundary value problem:

$$
\begin{aligned}
-\operatorname{div}(\mathbf{K} \nabla p(x)) & =f(x) \text { in } \Omega, \\
p(x) & =0 \quad \text { on } \partial \Omega,
\end{aligned}
$$

where $\Omega$ is a domain in $\mathbb{R}^{d}, d=2$ or 3 , and the boundary $\partial \Omega$ is polygonal and convex. The diffusion tensor $\mathbf{K}$ is assumed to be a uniformly symmetric positive definite matrix.

Defining the velocity $\mathbf{u}$ as

$$
\mathbf{u}=-\mathbf{K} \nabla p
$$

we can rewrite (1a) as a first order system for the unknowns $\mathbf{u}$ and $p$

$$
\begin{array}{r}
\mathbf{u}+\mathbf{K} \nabla p=0 \\
\operatorname{div}(\mathbf{u})=f .
\end{array}
$$

Note that in some application $\mathbf{K}$ can be discontinuous (heat transfer, reservoir simulation), although the normal component of $\mathbf{u}$ is continuous.

Problems (1) and (2) are equivalent. The weak formulation of problem (1) is: Find $(\mathbf{u}, p) \in \mathbf{U} \times P$ such that

$$
\begin{aligned}
\int_{\Omega} \mathbf{u} \cdot \mathbf{v}+\int_{\Omega} \mathbf{K} \nabla p \cdot \mathbf{v} & =\quad 0 \quad \forall \mathbf{v} \in \mathbf{V} \\
\int_{\Omega} \operatorname{div}(\mathbf{u}) q & =\int_{\Omega} f q \quad \forall q \in Q .
\end{aligned}
$$

Note that the test and trial spaces in (3) can be different. Similar methods have been considered in $[33,34]$ with particular choice

$$
\mathbf{U}=H(\operatorname{div}, \Omega), \quad P=H_{0}^{1}(\Omega), \quad \mathbf{V}=\left(L^{2}(\Omega)\right)^{d}, \quad Q=L^{2}(\Omega)
$$

where

$$
\begin{aligned}
H_{0}^{1}(\Omega) & =\left\{p \in L^{2}(\Omega), D p \in L^{2}(\Omega), p=0 \text { on } \partial \Omega\right\}, \\
H(\operatorname{div}, \Omega) & =\left\{\mathbf{v} \in\left(L^{2}(\Omega)\right)^{d}, \operatorname{div}(\mathbf{v}) \in L^{2}(\Omega)\right\} .
\end{aligned}
$$


It is generally difficult to find a stable pair discrete spaces $\left(\mathbf{U}_{h}, P_{h}\right), \mathbf{U}_{h} \subset H($ div,$\Omega), P_{h} \subset$ $H_{0}^{1}(\Omega)$ for unstructured meshes.

We would like to obtain a discrete method that replaces equation (3b) with an equation similar to the one derived from finite volume methods. Suppose the domain $\Omega$ is divided into set of control volumes $V_{i}, i=1, \ldots, n$, and assume $q$ is smooth enough. With integration by parts, we can rewrite $(3 \mathrm{~b})$ as

$$
\sum_{i=1}^{n}\left[\int_{\partial V_{i}} \mathbf{u} \cdot \mathbf{n} q-\int_{V_{i}} \nabla q \cdot \mathbf{u}\right]=\int_{\Omega} f q .
$$

Choosing $Q_{h}$ to be the space of piecewise constants on the control volumes, Eq. (4) becomes

$$
\sum_{i=1}^{n} \int_{\partial V_{i}} \mathbf{u}_{h} \cdot \mathbf{n} q=\int_{\Omega} f q,
$$

which is a typical equation in finite volume methods.

The equation (5) can be considered as an approximation of Eq. (3b) with non-conforming spaces $\mathbf{U}_{h}$ and $Q_{h}$.

\section{Discretization}

We introduce two different meshes that are "dual" to each other. The meaning of "dual" will become clear from the following example on which we build the discretization. In particular, the triangular (tetrahedral) mesh, e.g., the Delaunay triangulation $\mathcal{D}_{h}$, is used for the discretization of pressure $p$ (the triangles on Fig. 1). The velocity $\mathbf{u}$ is approximated on a control volume mesh built from the triangles, for example the Voronoi (PEBI) grid $\mathcal{V}_{h}$. One Voronoi control volume is depicted on Fig. 1 with dotted line.

We take the finite element methodology to construct the discretization. The first step is to replace the infinitely dimensional spaces $\mathbf{U}, P, \mathbf{V}$, and $Q$ in problem (3) with finite subspaces $\mathbf{U}_{h}, P_{h}, \mathbf{V}_{h}$, and $Q_{h}$. The subindex $h$ is used to emphasize the sequences of spaces for $h \rightarrow 0$, i.e., when the meshes become more refined, the dimension of the spaces increases. 


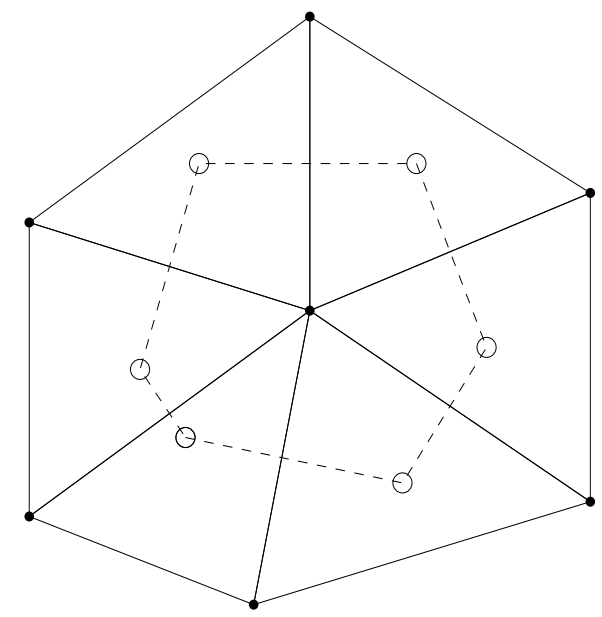

Figure 1: Voronoi box/Delaunay triangles

Denote with $\mathcal{E}_{h}$ the edges of control volumes in $\mathcal{V}_{h}$. We approximate the velocity u with a piecewise constant vector function that have continuous normal components on $\mathcal{E}_{h}$. More details will be given later on the construction of $\mathbf{U}_{h}$.

The discrete space $P_{h}$ of approximated pressure is defined on the triangulation $\mathcal{D}_{h}$. Every element $p \in P_{h}$ is a continuous piecewise linear function. We introduce a discrete subspace $Q_{h}$ of $L^{2}(\Omega)$ that consists of piecewise constants on control volumes $V \in \mathcal{V}_{h}$.

Then the discrete problem is defined as follows:

Find $\left(\mathbf{u}_{h}, p_{h}\right) \in \mathbf{U}_{h} \times P_{h}$ such that

$$
\begin{array}{r}
\int_{\Omega} \mathbf{u}_{h} \cdot \mathbf{v}_{h}+\int_{\Omega} \mathbf{K} \nabla p_{h} \cdot \mathbf{v}=\quad 0 \quad \forall \mathbf{v}_{h} \in \mathbf{U}_{h} \\
\sum_{V \in \mathcal{V}_{h}} \int_{\partial V} \mathbf{u}_{h} \cdot \mathbf{n} q_{h}=\int_{\Omega} f q_{h} \quad \forall q_{h} \in Q_{h} .
\end{array}
$$

Note that $\mathbf{U}_{h}=\mathbf{V}_{h}$.

The second step in the finite element discretization is to construct basis for $\mathbf{U}_{h}$.

\subsection{Basis of $\mathbf{U}_{h}$}

We would like to determine the velocity in such a way that the normal components on the edges of the control volumes in any given triangle are continuous. Consider a triangle $D=D_{i} \cup D_{j} \cup D_{k}$ as shown in Fig. 2. In each of the quadrilaterals $D_{i}, D_{j}$ and $D_{k}$ the velocity 


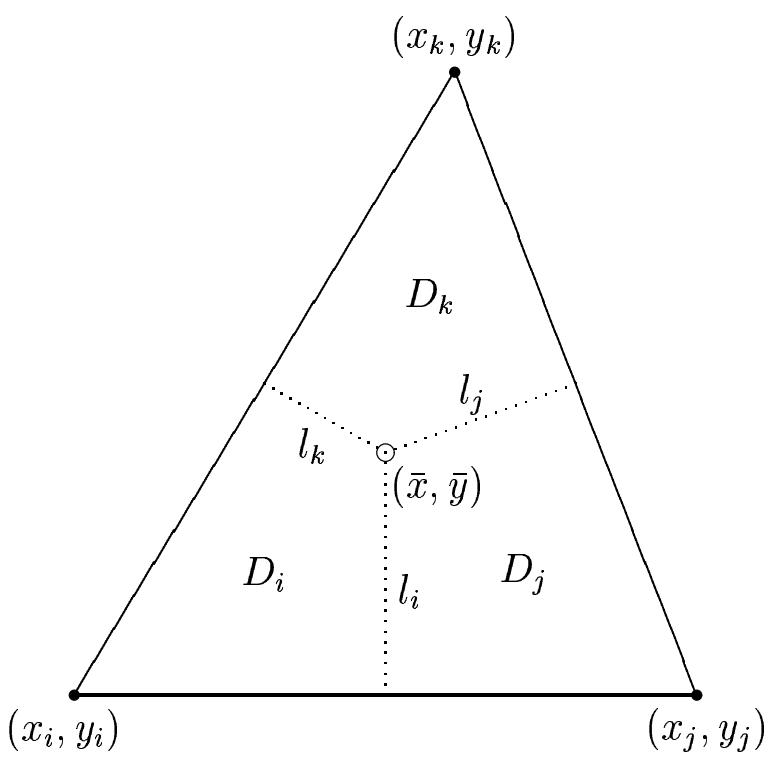

Figure 2: Triangle $D$

$\mathbf{v}$ is approximated with a constant function (six degrees of freedom). We additionally impose that the normal components on $l_{i}, l_{j}, l_{k}$ are continuous (subtract three degrees of freedom). Therefore, there are three degrees of freedom for the velocity space $\mathbf{U}_{h}$ in $D$. We choose them to be the integrals of the normal components on $l_{i}, l_{j}, l_{k}$, i.e., any vector function $\mathbf{v}_{\mid D} \in \mathbf{U}_{h}$ can be uniquely determined by the numbers

$$
v_{i}=\int_{l_{i}} \mathbf{v} \cdot \mathbf{n}_{i}, \quad v_{j}=\int_{l_{j}} \mathbf{v} \cdot \mathbf{n}_{j}, \quad v_{k}=\int_{l_{k}} \mathbf{v} \cdot \mathbf{n}_{k} .
$$

Note that the values of the velocity in one triangle are not directly connected with the values in the neighboring triangles.

Consider vector functions $\mathbf{e}_{i}, \mathbf{e}_{j}, \mathbf{e}_{k}$ defined with the relations

$$
\begin{aligned}
& \int_{l_{i}} \mathbf{e}_{i} \cdot \mathbf{n}_{i}=1, \quad \int_{l_{j}} \mathbf{e}_{i} \cdot \mathbf{n}_{j}=0, \quad \int_{l_{k}} \mathbf{e}_{i} \cdot \mathbf{n}_{k}=0, \\
& \int_{l_{i}} \mathbf{e}_{j} \cdot \mathbf{n}_{i}=0, \quad \int_{l_{j}} \mathbf{e}_{j} \cdot \mathbf{n}_{j}=1, \quad \int_{l_{k}} \mathbf{e}_{j} \cdot \mathbf{n}_{k}=0, \\
& \int_{l_{i}} \mathbf{e}_{k} \cdot \mathbf{n}_{i}=0, \quad \int_{l_{j}} \mathbf{e}_{k} \cdot \mathbf{n}_{j}=0, \quad \int_{l_{k}} \mathbf{e}_{k} \cdot \mathbf{n}_{k}=1 .
\end{aligned}
$$

It is easy to see that $\mathbf{e}_{i}, \mathbf{e}_{j}, \mathbf{e}_{k}$ are linearly independent and therefore form a basis. So $\mathbf{v}$ restricted to the triangle $D$ is equal to

$$
\mathbf{v}_{\mid D}=v_{i} \mathbf{e}_{i}+v_{j} \mathbf{e}_{j}+v_{k} \mathbf{e}_{k}
$$


Each of the vectors $\mathbf{e}_{i}, \mathbf{e}_{j}$ and $\mathbf{e}_{k}$, is defined on the whole triangle $D$, being piecewise constant on each of the quadrilaterals $D_{i}, D_{j}$ and $D_{k}$. One can compute the basis vectors once the normal vectors $\mathbf{n}_{i}, \mathbf{n}_{j}, \mathbf{n}_{k}$, and the lengths of the intervals $l_{i}, l_{j}, l_{k}$ are given. For example, $\mathbf{e}_{i}$ can be represented as

$$
\mathbf{e}_{i}=\left(\left(e_{1 \mid D_{i}}^{(i)}, e_{2 \mid D_{i}}^{(i)}\right),\left(e_{1 \mid D_{j}}^{(i)}, e_{2 \mid D_{j}}^{(i)}\right),\left(e_{1 \mid D_{k}}^{(i)}, e_{2 \mid D_{k}}^{(i)}\right)\right)
$$

According to the conditions (7a), in the quadrilateral $D_{i}$ we have the identities $\int_{l_{i}} \mathbf{e}_{i} \cdot \mathbf{n}_{i}=1$ and $\int_{l_{k}} \mathbf{e}_{i} \cdot \mathbf{n}_{k}=0$ that lead to the linear system for the unknown components

$$
\begin{aligned}
& e_{1 \mid D_{i}}^{(i)} n_{1}^{(i)}+e_{2 \mid D_{i}}^{(i)} n_{2}^{(i)}=1 /\left|l_{i}\right|, \\
& e_{1 \mid D_{i}}^{(i)} n_{1}^{(k)}+e_{2 \mid D_{i}}^{(i)} n_{2}^{(k)}=0 .
\end{aligned}
$$

Similarly we compute $e_{1 \mid D_{j}}^{(i)}$ and $e_{1 \mid D_{k}}^{(i)}$. Permuting appropriately the indexes $i, j$ and $k$ in the expressions above we can get the formulas for $\mathbf{e}_{j}$ and $\mathbf{e}_{k}$.

\subsection{Assembling}

The last step is to derive the actual finite volume scheme, in finite element terminology, to assemble the matrix. Consider Eq. (6a) with $\mathbf{v}_{h}=\mathbf{e}_{i}, \mathbf{e}_{j}$ and $\mathbf{e}_{k}$, i.e.,

$$
\begin{array}{r}
u_{1} \int_{D} \mathbf{e}_{i} \cdot \mathbf{e}_{i}+u_{2} \int_{D} \mathbf{e}_{j} \cdot \mathbf{e}_{i}+u_{3} \int_{D} \mathbf{e}_{k} \cdot \mathbf{e}_{i}+\int_{D} \mathbf{K} \nabla p \cdot \mathbf{e}_{i}=0, \\
u_{1} \int_{D} \mathbf{e}_{i} \cdot \mathbf{e}_{j}+u_{2} \int_{D} \mathbf{e}_{j} \cdot \mathbf{e}_{j}+u_{3} \int_{D} \mathbf{e}_{k} \cdot \mathbf{e}_{j}+\int_{D} \mathbf{K} \nabla p \cdot \mathbf{e}_{j}=0, \\
u_{1} \int_{D} \mathbf{e}_{i} \cdot \mathbf{e}_{k}+u_{2} \int_{D} \mathbf{e}_{j} \cdot \mathbf{e}_{k}+u_{3} \int_{D} \mathbf{e}_{k} \cdot \mathbf{e}_{k}+\int_{D} \mathbf{K} \nabla p \cdot \mathbf{e}_{k}=0 .
\end{array}
$$

Here we have taken into account that the restriction of $\mathbf{u}_{h}$ to the triangle $D$ is $\mathbf{u}_{h \mid D}=$ $u_{i} \mathbf{e}_{i}+u_{j} \mathbf{e}_{j}+u_{k} \mathbf{e}_{k}$. Note that the matrix $\left\{\mathbf{e}_{m} \cdot \mathbf{e}_{n}\right\}_{m, n=i, j, k}$ is symmetric and positive definite. Therefore, we can solve for the fluxes $u_{i}, u_{j}$ and $u_{k}$ and substitute them into Eq. (6b). Let

$$
t_{m n}=\int_{D} \mathbf{e}_{m} \cdot \mathbf{e}_{n},
$$

and denote the matrix

$$
T^{-1}=\left[\begin{array}{ccc}
t_{i i} & t_{j i} & t_{k i} \\
t_{i j} & t_{j j} & t_{k j} \\
t_{i k} & t_{j k} & t_{k k}
\end{array}\right]
$$


On the triangle $D$, we have

$$
p=p_{i} q_{i}+p_{j} q_{j}+p_{k} q_{k}
$$

where $q_{i}, q_{j}$ and $q_{k}$ are the basis "hat" functions. Denote

$$
m_{m n}=\int_{D} \mathbf{K} \nabla q_{m} \cdot \mathbf{e}_{n}
$$

and

$$
M=\left[\begin{array}{ccc}
m_{i i} & m_{j i} & m_{k i} \\
m_{i j} & m_{j j} & m_{k j} \\
m_{i k} & m_{j k} & m_{k k}
\end{array}\right]
$$

We call $M$ the mass matrix. Then

$$
\left[\begin{array}{l}
u_{1} \\
u_{2} \\
u_{3}
\end{array}\right]=-\left[\begin{array}{ccc}
t_{i i} & t_{j i} & t_{k i} \\
t_{i j} & t_{j j} & t_{k j} \\
t_{i k} & t_{j k} & t_{k k}
\end{array}\right]^{-1}\left[\begin{array}{ccc}
m_{i i} & m_{j i} & m_{k i} \\
m_{i j} & m_{j j} & m_{k j} \\
m_{i k} & m_{j k} & m_{k k}
\end{array}\right]\left[\begin{array}{c}
p_{i} \\
p_{j} \\
p_{k}
\end{array}\right]=-T M \mathbf{p} .
$$

Having expressed $\mathbf{u}_{h}$ through $p_{h}$, we obtain the final equation for each $p_{i}$ by substituting $\mathbf{u}_{h}$ into (6b) and testing with the basis of $Q_{h}$. Let $D_{1}, D_{2}, \ldots, D_{m}$ are the triangles in the support of $q_{i}$. Then the equation for $p_{i}$ is

$$
\sum_{V \in \mathcal{V}_{h}} \int_{\partial V}-T_{i} M_{i} \mathbf{p}_{i} \cdot \mathbf{n} q_{h}=\int_{\Omega} f q_{h}
$$

Here the subscript means $T_{i}, M_{i}$ and $\mathbf{p}_{i}$ are defined on triangle $D_{i}$.

Remark $\mathbf{1}$ The proposed method belongs to the class of "K methods" [18]. One can obtain a "K $\mathbf{K}^{-1}$ method" by first rewriting Eq. (2a) as

$$
\mathbf{K}^{-1} \mathbf{u}+\nabla p=0
$$

and then following the above procedure.

\section{Theoretical Framework}

In this section we state a theorem which is an easy generalization of the results by Nicolaides [26], Brezzi and Fortin [8] and Thomas and Trujillo [33, 34]. 
Take an abstract problem

$$
\begin{aligned}
\text { Find }(\mathbf{u}, p) & \in \mathbf{U} \times P \\
a(\mathbf{u}, \mathbf{v})+b(\mathbf{v}, p) & =<g, \mathbf{v}>\quad \forall \mathbf{v} \in \mathbf{V}, \\
c(\mathbf{u}, q) & =<f, q>\quad \forall q \in Q
\end{aligned}
$$

where $\left(\mathbf{U},\|\cdot\|_{\mathbf{U}}\right),\left(P,\|\cdot\|_{P}\right),\left(\mathbf{V},\|\cdot\|_{\mathbf{v}}\right)$ and $\left(Q,\|\cdot\|_{Q}\right)$ are four Hilbert spaces, $a(.,),. b(.,$.$) and$ $c(.,$.$) are bilinear forms defined respectively on \mathbf{U} \times \mathbf{V}, P \times \mathbf{V}$ and $\mathbf{U} \times Q$. The right hand sides are defined for $g \in \mathbf{V}^{\prime}, f \in Q^{\prime}$, where $\mathbf{V}^{\prime}$ and $Q^{\prime}$ are the dual spaces of $\mathbf{V}$ and $Q$ correspondingly.

Consider the discrete problem:

$$
\begin{aligned}
& \text { Find }\left(\mathbf{u}_{h}, p_{h}\right) \in \mathbf{U}_{h} \times P_{h} \\
& a_{h}\left(\mathbf{u}_{h}, \mathbf{v}_{\mathbf{h}}\right)+b_{h}\left(\mathbf{v}_{\mathbf{h}}, p_{h}\right)=<g, \mathbf{v}_{h}>_{h} \quad \forall \mathbf{v}_{h} \in \mathbf{V}_{h}, \\
& c_{h}\left(\mathbf{u}_{h}, q_{h}\right)=<f, q_{h}>_{h} \quad \forall q_{h} \in Q_{h},
\end{aligned}
$$

where $\left(\mathbf{U}_{h},\|\cdot\|_{\mathbf{U}_{h}}\right),\left(P_{h},\|\cdot\|_{P_{h}}\right),\left(\mathbf{V}_{h},\|\cdot\|_{\mathbf{v}_{h}}\right)$ and $\left(Q_{h},\|\cdot\|_{Q_{h}}\right)$ are four Hilbert spaces, $a_{h}(.,$.$) ,$ $b_{h}(.,$.$) and c_{h}(.,$.$) are bilinear forms defined respectively on \mathbf{U}_{h} \times \mathbf{V}_{h}, P_{h} \times \mathbf{V}_{h}$ and $\mathbf{U}_{h} \times Q_{h}$. Suppose that $\mathbf{U}_{h} \not \subset \mathbf{U}$ and $\mathbf{V}_{h} \not \subset \mathbf{V}$, i.e., discretization (9) is non-conforming.

Let $\mathbf{U}_{0 h}$ and $\mathbf{V}_{1 h}$ be the spaces defined by

$$
\begin{aligned}
& \mathbf{U}_{0 h}=\left\{\mathbf{u}_{h} \in \mathbf{U}_{h}, \quad \forall q_{h} \in Q_{h}, \quad c_{h}\left(\mathbf{u}_{h}, q_{h}\right)=0\right\} \\
& \mathbf{V}_{1 h}=\left\{\mathbf{v}_{h} \in \mathbf{V}_{h}, \quad \forall \mathbf{u}_{h} \in \mathbf{U}_{0 h}, \quad a_{h}\left(\mathbf{u}_{h}, \mathbf{v}_{h}\right)=0\right\}
\end{aligned}
$$

Assume that there exists three constants $A, B$ and $C$ independent of $h$ such that

$$
\begin{aligned}
& a_{h}\left(\mathbf{u}_{h}, \mathbf{v}_{h}\right) \leq A\left\|\mathbf{u}_{h}\right\|_{\mathbf{U}_{h}}\left\|\mathbf{v}_{h}\right\|_{\mathbf{v}_{h}}, \\
& b_{h}\left(\mathbf{v}_{h}, p_{h}\right) \leq B\left\|\mathbf{v}_{h}\right\|_{\mathbf{v}_{h}}\left\|p_{h}\right\|_{P_{h}} . \\
& c_{h}\left(\mathbf{u}_{h}, q_{h}\right) \leq C\left\|\mathbf{u}_{h}\right\|_{\mathbf{U}_{\mathbf{h}}}\left\|q_{h}\right\|_{Q_{h}}
\end{aligned}
$$

Then we have the following result: 
Theorem 1 Assume that the next three Babuška-Brezzi conditions are satisfied:

$$
\begin{aligned}
& \text { i) } \inf _{\mathbf{u}_{h} \in \mathbf{U}_{0 h}} \sup _{\mathbf{v}_{h} \in \mathbf{v}_{h}} \frac{a_{h}\left(\mathbf{u}_{h}, \mathbf{v}_{h}\right)}{\left\|\mathbf{u}_{h}\right\|_{\mathbf{U}_{h}}\left\|\mathbf{v}_{h}\right\|_{\mathbf{v}_{h}}} \geq \alpha, \\
& \text { ii) } \quad \inf _{p_{h} \in P_{h}} \sup _{\mathbf{v}_{h} \in \mathbf{V}_{1 h}} \frac{b_{h}\left(\mathbf{v}_{h}, p_{h}\right)}{\left\|\mathbf{v}_{h}\right\|_{\mathbf{v}_{h}}\left\|p_{h}\right\|_{P_{h}}} \geq \beta, \\
& \text { iii) } \quad \inf _{q_{h} \in Q_{h}} \sup _{\mathbf{u}_{h} \in \mathbf{U}_{h}} \frac{c_{h}\left(\mathbf{u}_{h}, q_{h}\right)}{\left\|\mathbf{u}_{h}\right\|_{\mathbf{U}_{h}}\left\|q_{h}\right\|_{Q_{h}}} \geq \gamma .
\end{aligned}
$$

and that

$$
\operatorname{dim}\left(\mathbf{U}_{h}\right)+\operatorname{dim}\left(P_{h}\right)=\operatorname{dim}\left(\mathbf{V}_{h}\right)+\operatorname{dim}\left(Q_{h}\right)
$$

Then problem (9) has a unique solution $\left(\mathbf{u}_{h}, p_{h}\right)$. Moreover, if $\alpha, \beta$ and $\gamma$ are independent of $h$, there exists positive constant $C$ independent of $h$ such that

$$
\begin{aligned}
& \left\|\mathbf{u}-\mathbf{u}_{h}\right\|_{\mathbf{U}_{h}}+\left\|p-p_{h}\right\|_{P_{h}} \\
& \quad \leq C\left(\inf _{\mathbf{v}_{h} \in \mathbf{V}_{h}}\left\|\mathbf{u}-\mathbf{v}_{h}\right\|_{\mathbf{v}_{h}}+\inf _{p_{h} \in P_{h}}\left\|p-p_{h}\right\|_{P_{h}}+M_{1 h}+M_{2 h}+M_{3 h}+M_{4 h}\right),
\end{aligned}
$$

where

$$
\begin{aligned}
& M_{1 h}=\sup _{\mathbf{v}_{h} \in \mathbf{V}_{h}} \frac{a_{h}\left(\mathbf{u}, \mathbf{v}_{h}\right)+b_{h}\left(\mathbf{v}_{h}, p\right)-<q, \mathbf{v}_{h}>}{\left\|\mathbf{v}_{h}\right\|_{\mathbf{v}_{h}}}, \\
& M_{2 h}=\sup _{\mathbf{v}_{h} \in \mathbf{V}_{h}} \frac{\left\langle g, \mathbf{v}_{h}>-<g, \mathbf{v}_{h}>_{h}\right.}{\left\|\mathbf{v}_{h}\right\|_{\mathbf{v}_{h}}}, \\
& M_{3 h}=\sup _{q_{h} \in q_{h}} \frac{c_{h}\left(\mathbf{u}, q_{h}\right)-<f, q_{h}>}{\left\|q_{h}\right\|_{Q_{h}}} \\
& M_{4 h}=\sup _{q_{h} \in Q_{h}} \frac{<f, q_{h}>-<f, q_{h}>_{h}}{\left\|q_{h}\right\|_{Q_{h}}} .
\end{aligned}
$$

\section{$5 \quad$ Stability and Error Estimates}

We start with several auxiliary results. The primary triangular mesh is assumed to be quasiregular as in the analysis of conforming finite element methods. The dual mesh, i.e., the control volumes, are formed by connecting the mass centers of the triangles to their edge midpoints. Some proofs are omitted for simplicity. 


\section{Lemma 1}

$$
\left\|\mathbf{u}_{h}\right\|_{0 . \Omega}^{2} \sim \sum_{E_{i j} \in \mathcal{E}_{h}}\left[\int_{E_{i j}} \mathbf{u}_{h} \cdot \mathbf{n}\right]^{2}, \quad \forall \mathbf{u}_{h} \in \mathbf{U}_{h}
$$

(The $\sim$ is use to indicate the equivalence of the norms.)

Proof: The proof follows from the definition of the space $\mathbf{U}_{h}$.

The error estimate for the constant interpolant in $\mathbf{V}_{h}$ is provided below.

\section{Lemma 2}

$$
\left\|\mathbf{v}-I_{h}^{c} \mathbf{v}\right\|_{0, \Omega} \leq C h\|\mathbf{v}\|_{1, \Omega}
$$

Proof: We follow the methodology developed by Ciarlet [13, Theorems 3.1.3-3.1.6, pp. 120-124]. Consider the reference element $\hat{D}$ and the constant interpolant $\hat{I}^{c}$ defined by

$$
\hat{I}^{c} \hat{\mathbf{v}}=\left[\int_{l_{i}} \hat{\mathbf{v}} \cdot \mathbf{n}_{i}\right] \mathbf{e}_{i}+\left[\int_{l_{j}} \hat{\mathbf{v}} \cdot \mathbf{n}_{j}\right] \mathbf{e}_{j}+\left[\int_{l_{k}} \hat{\mathbf{v}} \cdot \mathbf{n}_{k}\right] \mathbf{e}_{k}
$$

We have

$$
\begin{aligned}
\left\|\hat{I}^{c} \hat{\mathbf{v}}\right\|_{0, \hat{D}} & \leq C(\hat{D})\left(\left|\int_{l_{i}} \hat{\mathbf{v}} \cdot \mathbf{n}_{i}\right|\left|\mathbf{e}_{i}\right|+\left|\int_{l_{j}} \hat{\mathbf{v}} \cdot \mathbf{n}_{j}\right|\left|\mathbf{e}_{j}\right|+\left|\int_{l_{k}} \hat{\mathbf{v}} \cdot \mathbf{n}_{k}\right|\left|\mathbf{e}_{k}\right|\right) \\
& \leq C_{1}(\hat{D})\left(\|\hat{\mathbf{v}}\|_{0, l_{i}}+\|\hat{\mathbf{v}}\|_{0, l_{j}}+\|\hat{\mathbf{v}}\|_{0, l_{k}}\right) \\
& \leq C_{2}\|\hat{\mathbf{v}}\|_{1, \hat{D}} .
\end{aligned}
$$

In order to use Theorem 1 one has to specify the spaces and norms mentioned in it. We define the norms as

$$
\begin{aligned}
& \left\|p_{h}\right\|_{P_{h}}=\left\|p_{h}\right\|_{1, \Omega}, \quad\left\|q_{h}\right\|_{Q_{h}}=\left(\sum_{x_{i} \in \omega} \sum_{j \in \Pi(i)}\left(q_{i}-q_{j}\right)^{2}\right)^{1 / 2} \\
& \left\|\mathbf{u}_{h}\right\|_{\mathbf{U}_{h}}=\left\|\mathbf{u}_{h}\right\|_{\mathbf{v}_{h}}=\left\|\mathbf{u}_{h}\right\|_{0, \Omega}
\end{aligned}
$$

Recall a well known result for the equivalence of the norm on $P_{h}$ with $H_{0}^{1}$-seminorm [9]: 


\section{Lemma 3}

$$
\left\|q_{h}\right\| \sim\left|q_{h}\right|_{1, \Omega} \quad \forall q_{h} \in Q_{h} .
$$

The following theorem is the error estimate for the method proposed in this paper.

Theorem 2 Let $(\mathbf{u}, p)$ be the solution of problem (3) and $\left(\mathbf{u}_{h}, p_{h}\right)$ be the solution of (6). Then there exists a positive constant $C$ such that

$$
\left\|p-p_{h}\right\|_{1, \Omega}+\left\|\mathbf{u}-\mathbf{u}_{h}\right\|_{0, \Omega} \leq C h\left(|p|_{2, \Omega}+\|\mathbf{u}\|_{1, \Omega}\right) .
$$

Proof: It is easy to verify (10a) and (11a) for the bilinear form $a_{h}(.,$.$) .$

We check the conditions (10b) and (11b) for the bilinear form $b_{h}(.,$.$) as follows. Since \mathbf{K}$ is a uniformly positive definite matrix, there exists positive constants $k_{1}$ and $k_{2}$ such that

$$
k_{1} \xi^{t} \xi \leq \xi^{t} \mathbf{K} \xi \leq k_{2} \xi^{t} \xi, \quad \forall \xi \in \mathbf{R}^{d}
$$

So,

$$
b_{h}\left(\mathbf{v}_{h}, p_{h}\right)=\int_{\Omega} \mathbf{v}_{h} \cdot \mathbf{K} \nabla p_{h} \leq\left(\int_{\Omega} \mathbf{K} \mathbf{v}_{h} \cdot \mathbf{v}_{h}\right)^{1 / 2}\left(\int_{\Omega} \mathbf{K} \nabla p_{h} \cdot \nabla p_{h}\right)^{1 / 2} \leq k_{2}\left\|\mathbf{v}_{h}\right\|_{0, \Omega}\left\|p_{h}\right\|_{1, \Omega} .
$$

Choosing $\mathbf{v}_{h}\left(p_{h}\right)=\nabla p_{h}$. Then

$$
\begin{aligned}
b_{h}\left(\mathbf{v}_{h}\left(p_{h}\right), p_{h}\right) & =\int_{\Omega} \mathbf{v}_{h}\left(p_{h}\right) \cdot \mathbf{K} \nabla p_{h}=\left(\int_{\Omega} \mathbf{K} \mathbf{v}_{h}\left(p_{h}\right) \cdot \mathbf{v}_{h}\left(p_{h}\right)\right)^{1 / 2}\left(\int_{\Omega} \mathbf{K} \nabla p_{h} \cdot \nabla p_{h}\right)^{1 / 2} \\
& \geq k_{1}\left\|\mathbf{v}_{h}\left(p_{h}\right)\right\|_{0, \Omega}\left|p_{h}\right|_{1, \Omega} \geq C\left\|\mathbf{v}_{h}\left(p_{h}\right)\right\|_{0, \Omega}\left\|p_{h}\right\|_{1, \Omega} .
\end{aligned}
$$

Hence, in order to verify that (11b) is true, it suffices to show that $\nabla p_{h} \in \mathbf{V}_{1 h}, \forall p_{h} \in P_{h}$, i.e., we only need to show

$$
a_{h}\left(\mathbf{u}_{h}, \nabla p_{h}\right)=\sum_{D \in \mathcal{D}_{h}} \int_{D} \mathbf{u}_{h} \cdot \nabla p_{h}=0, \quad \forall \mathbf{u}_{h} \in \mathbf{U}_{0 h}, p_{h} \in P_{h} .
$$

Consider a triangle $D$ as shown in Fig. 2. Denote the normal vectors for $l_{i}, l_{j}, l_{k}$ as $\tilde{\mathbf{n}}_{i}, \tilde{\mathbf{n}}_{j}, \tilde{\mathbf{n}}_{k}$ with the direction $D_{i} \rightarrow D_{j} \rightarrow D_{k} \rightarrow D_{i}$. Note that $\tilde{\mathbf{n}}_{i}, \tilde{\mathbf{n}}_{j}, \tilde{\mathbf{n}}_{k}$ have length $\left|l_{i}\right|,\left|l_{j}\right|,\left|l_{k}\right|$ respectively, different from those normal vectors defined in Sec. 3.1. Let $s$ be the 
area of triangle $D$. Take $\mathbf{N}_{i}, \mathbf{N}_{j}$ and $\mathbf{N}_{k}$ to be the outwards normal vectors of triangle $D$. The usual convention applies, i.e., $\mathbf{N}_{i}$ is the normal vector of the edge $\left(x_{j}, y_{j}\right) \rightarrow\left(x_{k}, y_{k}\right)$, and the norm of $\mathbf{N}_{i}$ is equal to the length of the corresponding edge. Since the edge midpoints and mass center are used to form the control volumes, one can show

$$
\begin{aligned}
& \left|D_{i}\right|=\left|D_{j}\right|=\left|D_{k}\right|=\frac{s}{3}, \quad \tilde{\mathbf{n}}_{i}-\tilde{\mathbf{n}}_{j}+\tilde{\mathbf{n}}_{k}=0 \\
& \mathbf{N}_{i}=2\left(\tilde{\mathbf{n}}_{i}-\tilde{\mathbf{n}}_{k}\right), \quad \mathbf{N}_{j}=2\left(\tilde{\mathbf{n}}_{j}-\tilde{\mathbf{n}}_{i}\right), \quad \mathbf{N}_{k}=2\left(\tilde{\mathbf{n}}_{k}-\tilde{\mathbf{n}}_{j}\right) .
\end{aligned}
$$

Since $p_{h}$ is a linear function on $D$, we have

$$
\nabla p_{h}=-\frac{1}{2 s}\left(p_{i} \mathbf{N}_{i}+p_{j} \mathbf{N}_{j}+p_{k} \mathbf{N}_{k}\right)
$$

Therefore,

$$
\begin{aligned}
\int_{D} \mathbf{u}_{h} \cdot \nabla p_{h} & =\int_{D_{i}+D_{j}+D_{k}} \mathbf{u}_{h} \cdot \nabla p_{h} \\
& =-\frac{1}{3}\left(\left.\mathbf{u}_{h}\right|_{D_{i}}+\left.\mathbf{u}_{h}\right|_{D_{j}}+\left.\mathbf{u}_{h}\right|_{D_{k}}\right) \cdot\left(p_{i}\left(\tilde{\mathbf{n}}_{i}-\tilde{\mathbf{n}}_{k}\right)+p_{j}\left(\tilde{\mathbf{n}}_{j}-\tilde{\mathbf{n}}_{i}\right)+p_{k}\left(\tilde{\mathbf{n}}_{k}-\tilde{\mathbf{n}}_{j}\right)\right),
\end{aligned}
$$

where the coefficient of $\left(-\frac{1}{3} p_{i}\right)$ is equal to

$$
\begin{aligned}
\left(\left.\mathbf{u}_{h}\right|_{D_{i}}+\left.\mathbf{u}_{h}\right|_{D_{j}}+\left.\mathbf{u}_{h}\right|_{D_{k}}\right) \cdot\left(\tilde{\mathbf{n}}_{i}-\tilde{\mathbf{n}}_{k}\right) \\
\quad=\left.\mathbf{u}_{h}\right|_{D_{i}} \cdot\left(\tilde{\mathbf{n}}_{i}-\tilde{\mathbf{n}}_{k}\right)+\left.\mathbf{u}_{h}\right|_{D_{j}} \cdot\left(\tilde{\mathbf{n}}_{i}-\tilde{\mathbf{n}}_{k}\right)+\left.\mathbf{u}_{h}\right|_{D_{k}} \cdot\left(\tilde{\mathbf{n}}_{i}-\tilde{\mathbf{n}}_{k}\right) \\
\quad=\left.\mathbf{u}_{h}\right|_{D_{i}} \cdot\left(\tilde{\mathbf{n}}_{i}-\tilde{\mathbf{n}}_{k}\right)+\left.\mathbf{u}_{h}\right|_{D_{j}} \cdot\left(2 \tilde{\mathbf{n}}_{i}-\tilde{\mathbf{n}}_{j}\right)+\left.\mathbf{u}_{h}\right|_{D_{k}} \cdot\left(\tilde{\mathbf{n}}_{j}-2 \tilde{\mathbf{n}}_{k}\right) \\
\quad=\left.\mathbf{u}_{h}\right|_{D_{i}} \cdot\left(\tilde{\mathbf{n}}_{i}-\tilde{\mathbf{n}}_{k}\right)+\left.2 \mathbf{u}_{h}\right|_{D_{j}} \cdot \tilde{\mathbf{n}}_{i}-\left.\mathbf{u}_{h}\right|_{D_{j}} \cdot \tilde{\mathbf{n}}_{j}+\left.\mathbf{u}_{h}\right|_{D_{k}} \cdot \tilde{\mathbf{n}}_{j}-\left.2 \mathbf{u}_{h}\right|_{D_{k}} \cdot \tilde{\mathbf{n}}_{k} \\
\quad=\left.\mathbf{u}_{h}\right|_{D_{i}} \cdot\left(\tilde{\mathbf{n}}_{i}-\tilde{\mathbf{n}}_{k}\right)+\left.2 \mathbf{u}_{h}\right|_{D_{i}} \cdot \tilde{\mathbf{n}}_{i}-\left.\mathbf{u}_{h}\right|_{D_{j}} \cdot \tilde{\mathbf{n}}_{j}+\left.\mathbf{u}_{h}\right|_{D_{j}} \cdot \tilde{\mathbf{n}}_{j}-\left.2 \mathbf{u}_{h}\right|_{D_{i}} \cdot \tilde{\mathbf{n}}_{k} \\
\quad=\left.3 \mathbf{u}_{h}\right|_{D_{i}} \cdot\left(\tilde{\mathbf{n}}_{i}-\tilde{\mathbf{n}}_{k}\right) .
\end{aligned}
$$

In the above equalities, we use the fact that the normal component of $u_{h}$ is continuous across the edges $l_{i}, l_{j}, l_{k}$. So,

$$
\int_{D} \mathbf{u}_{h} \cdot \nabla p_{h}=-\left(\left.p_{i} \mathbf{u}_{h}\right|_{D_{i}} \cdot\left(\tilde{\mathbf{n}}_{i}-\tilde{\mathbf{n}}_{k}\right)+\left.p_{j} \mathbf{u}_{h}\right|_{D_{j}} \cdot\left(\tilde{\mathbf{n}}_{j}-\tilde{\mathbf{n}}_{i}\right)+\left.p_{k} \mathbf{u}_{h}\right|_{D_{k}} \cdot\left(\tilde{\mathbf{n}}_{k}-\tilde{\mathbf{n}}_{j}\right)\right) \cdot
$$


Substitute it into Eq. (13) and sum all the coefficients of $p_{i}$. Then,

$$
a_{h}\left(\mathbf{u}_{h}, \nabla p_{h}\right)=\sum_{V \in \mathcal{V}_{h}} \int_{\partial V} \mathbf{u}_{h} \cdot \mathbf{n} p_{V}=0,
$$

where $p_{V}$ denotes the value of $p_{h}$ at the 'center' of control volume $V$ and the last equality follows from the fact $\mathbf{u}_{h} \in \mathbf{U}_{0 h}$.

Now we check the conditions (10c) and (11c). The condition (10c) can be easily verified. Given $q_{h} \in Q_{h}$, construct $\mathbf{u}\left(q_{h}\right)$ such that $\mathbf{u}\left(q_{h}\right) \cdot \mathbf{n}_{\mid E_{i j}}\left|E_{i j}\right|=q_{i}-q_{j}$. Then

$$
\begin{aligned}
c_{h}\left(\mathbf{u}\left(q_{h}\right), q_{h}\right) & =\sum_{V \in \mathcal{V}_{h}} \int_{\partial V} \mathbf{u}\left(q_{h}\right) \cdot \mathbf{n} q_{h}=\sum_{E_{i j} \in \mathcal{E}_{h}} \mathbf{u}\left(q_{h}\right) \cdot \mathbf{n}_{\mid E_{i j}}\left|E_{i j}\right|\left(q_{i}-q_{j}\right) \\
& =\left(\sum_{E_{i j} \in \mathcal{E}_{h}}\left[\int_{E_{i j}} \mathbf{u}\left(q_{h}\right) \cdot \mathbf{n}\right]^{2}\right)^{1 / 2}\left(\sum_{E_{i j} \in \mathcal{E}_{h}}\left(q_{i}-q_{j}\right)^{2}\right)^{1 / 2} \\
& \geq C\left\|\mathbf{u}\left(q_{h}\right)\right\|_{0, \Omega}\left\|q_{h}\right\|_{1, \Omega} .
\end{aligned}
$$

Therefore,

$$
\sup _{\mathbf{u} \in \mathbf{U}_{h}} \frac{c_{h}\left(\mathbf{u}, q_{h}\right)}{\|\mathbf{u}\|_{0, \Omega}} \geq \frac{c_{h}\left(\mathbf{u}\left(q_{h}\right), q_{h}\right)}{\left\|\mathbf{u}\left(q_{h}\right)\right\|_{0, \Omega}} \geq C\left\|q_{h}\right\|_{1, \Omega} .
$$

To finish the proof of the theorem we note that $M_{1 h}, M_{2 h}, M_{3 h}$ and $M_{4 h}$ are equal to zero for the corresponding bilinear forms and Lemma 2 provides a bound for the interpolation of $\mathbf{u}$ in (12).

\section{$6 \quad$ Numerical Results}

In this section we present numerical experiments with three model problems on a domain $\Omega$ - a pentagon with a square hole inside. The coordinates of the pentagon vertexes are $((-1,0),(1,0),(1.6,2),(0,4),(-1.6,2))$ and the coordinates of the square hole are $((-0.7,0.9),(0.7,0.9),(0.7,2.3),(-0.7,2.3))$. The domain $\Omega$ is shown on Fig. 3 with 66 mesh points and 92 triangles. We choose such a domain to illustrate the flexibility of the finite volume method.

The Delaunay triangulations are generated using the software product triangle developed by J. R. Shewchuk [32]. Triangle is a Delaunay triangulator and it provides the control 


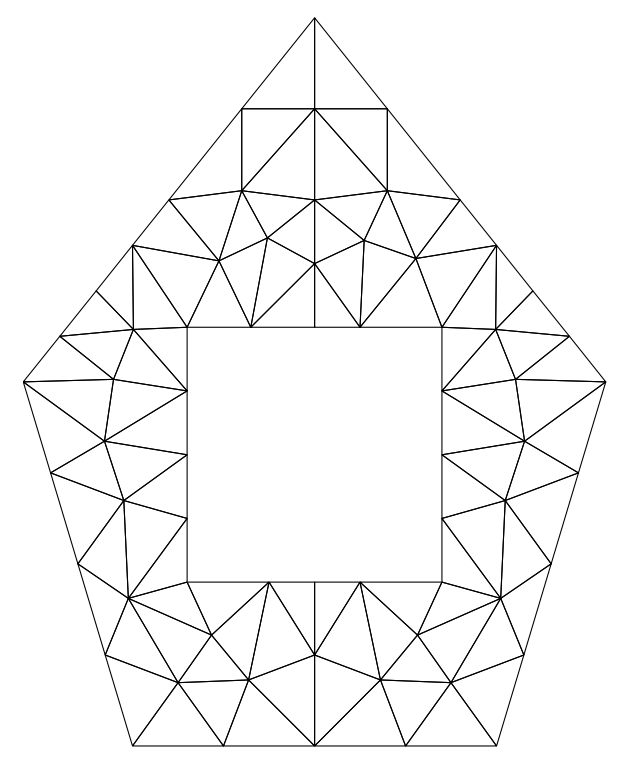

Figure 3: Domain $\Omega$ and mesh points

on the maximum area of the triangles. We used this option to generate five Delaunay triangulations. The maximum area of the triangles decreases four times on every successive level. The number of nodes increase roughly by four. The control volumes are created by connecting the center of gravity of each triangle to the center of its edges.

Problem 1 (Laplacian) Take

$$
\mathbf{K}(\mathrm{x})=I
$$

and the exact solution

$$
p(x, y)=\sin (\pi x) \sin (\pi y)
$$

Problem 2 (Smooth full tensor (see Shashkov [31])) Take

$$
\mathbf{K}(x, y)=R D(x, y) R^{T}
$$

where

$$
R=\left[\begin{array}{cc}
\cos (\phi) & -\sin (\phi) \\
\sin (\phi) & \cos (\phi)
\end{array}\right], \quad D(x, y)=\left[\begin{array}{cc}
d_{1} & 0 \\
0 & d_{2}
\end{array}\right]
$$


and

$$
\begin{aligned}
\phi & =\frac{\pi}{4}, \\
d_{1} & =1+2 x^{2}+y^{2}+y^{5}, \\
d_{2} & =1+x^{2}+2 y^{2}+x^{3},
\end{aligned}
$$

and the exact solution is

$$
p(x, y)=\sin (\pi x) \sin (\pi y)
$$

\section{Problem 3 (Discontinuous full tensor) Let}

$$
\mathbf{K}(x, y)= \begin{cases}{\left[\begin{array}{ll}
2 & 1 \\
1 & 2
\end{array}\right]} & \text { for } x \leq 0 \\
{\left[\begin{array}{ll}
1 & 0 \\
0 & 1
\end{array}\right]} & \text { for } x>0\end{cases}
$$

The exact solution is given as

$$
p(x, y)= \begin{cases}(1+x) y, & \text { for } x \leq 0 \\ (1+x) y+x(1+y), & \text { for } x>0 .\end{cases}
$$

We compute the $L^{2}$ error of $p_{h}$ and $\mathbf{u}_{h}$, the $H^{1}$ error of $p_{h}$, and the $H(d i v)_{h}$ error for $\mathbf{u}_{h}$. In particular, the $H(d i v)_{h}$ error takes the form

$$
\left\|\mathbf{u}-\mathbf{u}_{h}\right\|_{H(d i v), h}=\left(\sum_{K_{i} \in \mathcal{F}_{h}} \int_{\partial K_{i}}\left(\mathbf{u}_{h} \cdot \mathbf{n}+\mathbf{K} \nabla p \cdot \mathbf{n}\right)^{2} d l\right)^{1 / 2} .
$$

The numerical results reported in Tab. 1-3 confirm the first order error estimates for $p_{h}$ and $\mathbf{u}_{h}$ proven in Theorem 2. We also observe that there is second order convergence of the pressure in $L^{2}$-norm and a half order convergence for the discrete divergence of the velocity.

Remark 2 Similar results are obtained for all the three test problems with the "K $\mathbf{K}^{-1}$ " method discussed in Remark 1. 
Table 1: Discrete norms of the error for Problem 1

\begin{tabular}{|c|c|c|c|c|}
\hline$N$ & $\left\|p-p_{h}\right\|_{0, \Omega}$ & $\left\|p-p_{h}\right\|_{1, \Omega}$ & $\left\|\mathbf{u}-\mathbf{u}_{h}\right\|_{0, \Omega}$ & $\left\|\mathbf{u}-\mathbf{u}_{h}\right\|_{H(\text { div }), h}$ \\
\hline 66 & $3.127 .10^{-1}$ & $2.067 .10^{-0}$ & $2.603 .10^{-0}$ & $2.891 .10^{-0}$ \\
\hline 233 & $7.319 .10^{-2}$ & $9.070 .10^{-1}$ & $1.210 .10^{-0}$ & $1.700 .10^{-0}$ \\
\hline 878 & $1.815 .10^{-2}$ & $4.440 .10^{-1}$ & $5.973 .10^{-1}$ & $1.112 .10^{-0}$ \\
\hline 3353 & $4.466 .10^{-3}$ & $2.160 .10^{-1}$ & $2.928 .10^{-1}$ & $7.609 .10^{-1}$ \\
\hline 13105 & $1.124 .10^{-3}$ & $1.070 .10^{-1}$ & $1.453 .10^{-1}$ & $5.303 .10^{-1}$ \\
\hline \hline Order & 2.121 & 1.110 & 1.085 & 0.633 \\
\hline
\end{tabular}

Table 2: Discrete norms of the error for Problem 2

\begin{tabular}{|c|c|c|c|c|}
\hline$N$ & $\left\|p-p_{h}\right\|_{0, \Omega}$ & $\left\|p-p_{h}\right\|_{1, \Omega}$ & $\left\|\mathbf{u}-\mathbf{u}_{h}\right\|_{0, \Omega}$ & $\left\|\mathbf{u}-\mathbf{u}_{h}\right\|_{H(d i v), h}$ \\
\hline 66 & $3.847 .10^{-1}$ & $2.493 .10^{-0}$ & $2.776 .10^{+2}$ & $3.614 .10^{+2}$ \\
\hline 233 & $9.474 .10^{-2}$ & $9.802 .10^{-1}$ & $1.575 .10^{+2}$ & $2.621 .10^{+2}$ \\
\hline 878 & $2.513 .10^{-2}$ & $4.797 .10^{-1}$ & $7.894 .10^{+1}$ & $1.631 .10^{+2}$ \\
\hline 3353 & $5.892 .10^{-3}$ & $2.266 .10^{-1}$ & $4.001 .10^{+1}$ & $1.146 .10^{+2}$ \\
\hline 13105 & $1.698 .10^{-3}$ & $1.120 .10^{-1}$ & $1.785 .10^{+1}$ & $7.764 .10^{+1}$ \\
\hline \hline Order & 2.056 & 1.157 & 1.036 & 0.589 \\
\hline
\end{tabular}

Table 3: Discrete norms of the error for Problem 3

\begin{tabular}{|c|c|c|c|c|}
\hline$N$ & $\left\|p-p_{h}\right\|_{0, \Omega}$ & $\left\|p-p_{h}\right\|_{1, \Omega}$ & $\left\|\mathbf{u}-\mathbf{u}_{h}\right\|_{0, \Omega}$ & $\left\|\mathbf{u}-\mathbf{u}_{h}\right\|_{H(\text { div }),}$ \\
\hline 66 & $4.862 .10^{-2}$ & $5.756 .10^{-1}$ & $8.721 .10^{-1}$ & $9.048 .10^{-1}$ \\
\hline 233 & $9.450 .10^{-3}$ & $2.577 .10^{-1}$ & $4.193 .10^{-1}$ & $5.906 .10^{-1}$ \\
\hline 878 & $2.609 .10^{-3}$ & $1.298 .10^{-1}$ & $2.073 .10^{-1}$ & $4.075 .10^{-1}$ \\
\hline 3353 & $5.818 .10^{-4}$ & $6.339 .10^{-2}$ & $1.028 .10^{-1}$ & $2.806 .10^{-1}$ \\
\hline 13105 & $1.603 .10^{-5}$ & $3.155 .10^{-2}$ & $5.103 .10^{-2}$ & $1.959 .10^{-1}$ \\
\hline \hline Order & 2.145 & 1.088 & 1.069 & 0.574 \\
\hline
\end{tabular}




\section{Conclusion}

We have developed a locally conservative and flux-continuous "K method" for the elliptic diffusion equation with anisotropic and heterogeneous diffusion tensor. The method is well defined on very general meshes and is directly applicable to 3-D problems. Stability and error estimate are also established when the control volumes are formed by connecting the mass center of the triangles to their edge midpoints. Numerical experiments confirm our theoretical results. Similar numerical results were obtained for its corresponding " $\mathbf{K}^{-1}$ method".

There are still several theoretical and practical issues left unresolved like the convergence of the pressure in $L^{2}$-norm and convergence of the velocity in the discrete $H(d i v)$-norm.

\section{References}

[1] I. Aavatsmark, T. Barkve, O. Be, and T. Mannseth. Discretization on unstructured grids for inhomogeneous, anisotropic media. Part I: Derivation of the methods. SIAM J. Sci. Stat. Comput., 19:1700-1716, 1998.

[2] L. Angermann. An introduction to finite volume methods for linear elliptic equations of second order. Technical Report No. 163, Friedrich-Alexander University of ErlangenNuremberg, Erlangen, 1995.

[3] T. Arbogast, C. N. Dawson, P. T. Keenan, M. F. Wheeler, and I. Yotov. Enhanced cell-centered finite differences for elliptic operators on general geometry. SIAM J.Sci. Comput., 19(2):404-425, 1998.

[4] T. Arbogast, M. F. Wheeler, and I. Yotov. Mixed finite elements for elliptic problems with tensor coefficients as cell-centered finite differences. SIAM J.Numer. Anal., 34(2):828-853, 1997.

[5] R. E. Bank and D. J. Rose. Some error estimates for the box method. SIAM J.Numer. Anal., 24:777-787, 1987. 
[6] M. Berndt, K. Lipnukov, J. Moulton, and M. Shashkov. Convergence of mimetic finite difference discretizations of the diffusion equation. East-West J. Numer. Math., 9:253$316,2001$.

[7] M. Berndt, K. Lipnukov, M. Shashkov, M. Wheeler, and I. Yotov. Superconvergence of the velocity in mimetic finite difference methods on quadrilaterals. SIAM J. Numer. Anal., 2005, to appear.

[8] F. Brezzi and M. Fortin. Mixed and Hybrid Finite Element Methods. Springer-Verlag, New York, 1991.

[9] Z. Cai. On the finite volume element method. Numer. Math., 58:713-735, 1991.

[10] Z. Cai, J. Jones, , S. F. McCormick, and T. Russell. Control volume mixed finite element methods. Computational Geosciences, 1:289-315, 1997.

[11] Z. Cai, J. Mandel, and S. F. McCormick. The finite volume element method for diffusion equations on general triangulations. SIAM J.Numer. Anal., 28:392-402, 1991.

[12] S.-H. Chou, D. Y. Kwak, and K. Y. Kim. A general framework for constructing and analyzing mixed finite volume methods on quadrilateral grids: The overlapping covolume case. SIAM J. Numer. Anal., 39(4):1170-1196, 2001.

[13] P. G. Ciarlet. The Finite Element Method for Elliptic Problems. North-Holland Publishers, Amsterdam, 1978.

[14] M. G. Edwards. Symmetric, flux continuous, positive definite approximation of the elliptic full tensor for pressure equation in local conservation form. In C. et. al., editor, Proceedings of 13th SPE Reservoir Simulation Symposium, San Antonio, Texas, USA (SPE 29147), 1995.

[15] W. Hackbusch. On first and second order box schemes. Computing, 41:277-296, 1989. 
[16] J. Huang and S. Xi. On the finite volume element method for general self-adjoint elliptic problem. SIAM J.Numer. Anal., 35(5):1762-1774, 1998.

[17] T. Kerkhoven. Piecewise linear Petrov-Galerkin error estimates for the box method. SIAM J.Numer. Anal., 33(5):1864-1884, 1996.

[18] R. Klausen and T. Russell. Relationships among some locally conservative discretization methods which handle discontinuous coefficients. Computational Geosciences, submitted.

[19] R. Klausen and R. Winther. Convergence of multipoint flux approximations on quadrilateral grids. SIAM J. Numer. Anal., submitted.

[20] Y. Kuznetsov and S. Repin. New mixed finite element method on polygonal and polyhedral meshes. Russian Journal of Numerical Analysis and Mathematical Modeling, 18(3):261-278, 2003.

[21] R. D. Lazarov and I. D. Mishev. Finite volume methods for reaction-diffusion problems. In F. Benkhaldoun and R. Vilsmeier, editors, Finite Volumes for Complex Applications, pages 231-240. Hermes, 1996.

[22] T. Mathew and J. Wang. Mixed finite element methods over quadrilaterals. In I. T. Dimov, B. Sendov, and P. Vassilevski, editors, Proc. 3d Internat. Conf. on Advances in Numerical Methods and Applications, pages 203-214, Singapore, 1994. World Scientific.

[23] I. D. Mishev. Finite volume methods on Voronoi meshes. Numer. Methods Partial Differential Eq., 14:193-212, 1998.

[24] I. D. Mishev. Finite volume element methods for non-definite problems. Numer. Math., 83:161-175, 1999.

[25] R. L. Naff, T. F. Russell, and J. D. Wilson. Shape functions for velocity interpolation in general hexahedral cells. Computational Geosciences, 6(3-4):285-314, 2002. 
[26] R. A. Nicolaides. Existence, uniqueness and approximation for generalized saddle appoint problems. SIAM J.Numer. Anal., 19(2):349-357, 1982.

[27] P. A. Raviart and J. M. Thomas. A mixed finite element methodd for 2nd order elliptic problems. In Mathematical aspects of the Finite Element Methods, Lecture Notes in Math. Springer-Verlag, Berlin, 1977.

[28] J. E. Roberts and J. M. Thomas. Mixed and hybrid methods. In P. G. Ciarlet and J. L. Lions, editors, Handbook of Numerical Analysis, volume 2, pages 524-639. Elsevier Science Publisher B.V., 1991.

[29] R.Vanselow and H. P. Scheffler. Convergence analysis of a finite volume method via a new non-conforming finite element method. Numer. Methods Partial Differential Eq., 14:213-231, 1998.

[30] T. Schmidt. Box schemes on quadrilateral meshes. Computing, 51:271-292, 1993.

[31] M. Shashkov. Conservative Finite-difference methods on general grids. CRC Press, New York, 1996.

[32] J. R. Shewchuk. Triangle: Engineering a 2D quality mesh generator and Delaunay triangulator. In Proc. First Workshop on Applied Computational Geometry, Philadelphia, Pennsylvania, pages 124-133, ACM, 1996.

[33] J. M. Thomas and D. Trujillo. Analysis of finite volume methods. Technical Report 19, Universite de Pau et des Pays de L'adour, Pau, France, 1995.

[34] J. M. Thomas and D. Trujillo. Convergence of finite volume methods. Technical Report 20, Universite de Pau et des Pays de L'adour, Pau, France, 1995.

[35] S. K. Verma. Flexible grids for reservoir simulations. PhD thesis, Stanford University, California, 1996. 